\title{
Correlation Analysis of circRNA Circ_0071662 in Diagnosis and Prognosis of Esophageal Squamous Cell Carcinoma
}

\author{
Lei Xia' \\ Wei Liang ${ }^{2}$ \\ Dan Que 2 \\ Qichao Xie ${ }^{2}$ \\ 'Cancer Center, The Second Affiliated \\ Hospital of Chongqing Medical University, \\ Chongqing City, 400000, People's \\ Republic of China; ${ }^{2}$ Department of \\ Oncology, The Third Affiliated Hospital \\ of Chongqing Medical University, \\ Chongqing City, 400000, People's \\ Republic of China
}

Background: The role of circRNA circ_0071662 has been studied in bladder cancer. The present study aimed to analyze its involvement in esophageal squamous cell carcinoma (ESCC).

Methods: Patients with ESCC $(n=66)$, esophageal ulcer $(E U, n=66)$, or gastroesophageal reflux disease (GERD, $n=66)$ and healthy controls $(n=66)$ were enrolled in this study. Plasma samples were collected from all patients and controls. ESCC and paired non-tumor tissue samples were collected from ESCC patients. Circ_0071662 levels in these samples were determined by RT-qPCRs. Diagnostic and prognostic values of circ_0071662 for ESCC were analyzed with ROC curve and survival curve analyses.

Results: Circ_0071662 level was decreased in ESCC, but not in GERN and EU compared to the controls and in ESCC tissues compared to the non-tumor tissues. Plasma circ_0071662 was closely correlated with patients' tumor size but not with other clinical features. Decreased plasma circ_0071662 levels separated ESCC patients from GERN patients, EU patients, and healthy controls. Low plasma circ_0071662 levels were closely correlated with worse survival outcomes of ESCC patients.

Conclusion: Circ_0071662 is lowly expressed in ESCC and may serve as a potential diagnostic and prognostic biomarker for ESCC.

Keywords: esophageal squamous cell carcinoma, circ_0071662, diagnosis, prognosis

\section{Introduction}

Esophageal squamous cell carcinoma (ESCC) originates from the flat and thin cells lining the inside of the esophagus. ${ }^{1}$ ESCC accounts for more than $90 \%$ of esophageal cancer, the sixth most common cancer worldwide. ${ }^{1}$ ESCC is most frequently observed in the middle and upper part of the esophagus but can also affect other parts of the esophagus. ${ }^{2,3}$ ESCC patients, in general, have poor survival, although their overall survival is significantly affected by stages. ${ }^{4}$ It is reported that about $47 \%$ of patients with ESCC detected at the local stage can survive longer than 5 years, while only $25 \%$ of ESCC patients with regional metastasis and $5 \%$ of ESCC patients with distant metastasis can survive 5 years. ${ }^{5,6}$ Therefore, the key for the survival of ESCC patients is still early diagnosis. ${ }^{7}$

One of the major causes of delayed diagnosis of ESCC is misdiagnosis. ${ }^{8,9}$ Several clinical disorders, such as esophageal ulcer (EU) and gastroesophageal reflux disease (GERD can mimic the symptoms of ESCC, ${ }^{10,11}$ leading to common misdiagnosis. Although different clinical disorders may share similar clinical
Correspondence: Qichao Xie

Tel +86 23-60353465

Email qichaoxieyubei@I63.com 
presentations, they may require the involvement of different molecular factors. Therefore, certain differentially expressed factors may serve as biomarkers to distinguish ESCC from other clinical disorders. ${ }^{12,13}$ CircRNAs encode no protein products, but they are involved in the regulation of protein synthesis and degradation, thereby exerting oncogenic or tumor-suppressive functions. ${ }^{14}$ Therefore, circRNAs may be applied as the diagnostic biomarkers for ESCC. The role of circRNA circ_0071662 has been studied in bladder cancer. ${ }^{15}$ The present study aimed to analyze its involvement in ESCC.

\section{Materials and Methods}

\section{Participants and a 5-Year Follow-Up}

The study was approved by the Ethics Committee of the Third Affiliated Hospital of Chongqing Medical University and included 66 ESCC patients (43 males and 23 females, $55.9 \pm 7.5$ years), 66 esophageal ulcer (EU) patients (43 males and 23 females, 55.7 7.1 years), 66 gastroesophageal reflux disease (GERD) patients (43 males and 23 females, $55.8 \pm 7.4$ years) and 66 healthy controls (43 males and 23 females, $55.8 \pm 8.0$ years) who admitted to the hospital. All ESCC patients were diagnosed by multiple approaches, including imaging techniques and biopsies. EU and GERD patients were diagnosed according to the standard method. The inclusion criteria were 1) newly diagnosed cases and 2) no therapies had been initiated. The exclusion criteria were 1) patients with blood relationships, 2) patients complicated with severe infections and other severe diseases, and 3) pregnant women. All healthy controls were enrolled if they completed systemic physiological examination including height, weight, vision, blood routine, urine routine, stool routine, lung function test, liver and kidney function test, blood pressure test, blood sugar and blood lipid test and electrocardiography and had normal physiological functions. All participants signed informed consent. From the day of admission, the 66 ESCC patients were visited monthly via telephone or outpatients visit to monitor their survival. All 66 patients completed the follow-up or died of ESCC during the follow-up. None of these patients died of other causes.

\section{Preparation of Tissue and Plasma Samples} After admission, paired non-tumor and ESCC tumor specimens were collected from all 66 ESCC patients either through biopsy or dissecting the resected primary tumors. Blood $(3 \mathrm{~mL})$ samples were extracted from the elbow vein of both patients (ESCC, EU, and GERD) and healthy controls after fasted overnight and used to isolate plasma samples using conventional methods.

\section{RNA Extraction and Purification}

RNA samples were immediately isolated from the collected samples using Monarch Total RNA Miniprep Kit (T2010, NEB). In brief, samples were lysed, loaded onto gDNA removal columns, and centrifugation at $12000 \mathrm{~g}$ for $10 \mathrm{~min}$. The follow-through RNA samples were then loaded onto RNA purification columns, treated with DNase I, washed with washing buffer, and eluted with RNase-free water. Finally, RNA samples were subjected to the analysis of quality and quantity using 2100 Bioanalyzer.

\section{RT-qPCRs}

RNA samples with a RIN value higher than 8.5 were diluted to about $1500 \mathrm{ng} / \mu \mathrm{L}$ with RNase-free water. After that, $4 \mu \mathrm{L}$ RNA samples were used to prepare cDNA samples through reverse transcriptions using a High-Capacity cDNA Reverse Transcription Kit (Applied Biosystems). Circ_0071662 levels were determined by qPCRs with $18 \mathrm{~S}$ rRNA as the internal control using Fast Start Universal SYBR Green Master (Roche). Relative gene expression levels were normalized using the $2^{-\Delta \Delta \mathrm{Ct}}$ method.

\section{Statistical Analysis}

SPSS software program (15.0) was applied to analyze data and plot images. With ESCC patients as the true positive cases, and EU, GERD or controls as the true negative cases, ROC curve analysis was carried out to analyze the role of plasma circ_0071662 in the diagnosis of ESCC. The 66 ESCC patients were grouped into high and low plasma circ_0071662 level groups $(n=33)$ with the median plasma circ_0071662 level in ESCC patients as the cutoff value, and chi-squared test was applied to explore the associations between plasma circ_0071662 and patients' clinical factors. The survival curves of patients in both circ_0071662 level groups were plotted based on the 5-year survival data and compared using Log rank test. $\mathrm{P}<0.05$ was statistically significant.

\section{Results}

\section{RT-qPCR Analysis of Circ_007I662 Levels in Tissues and Plasma}

Circ_0071662 levels in paired ESCC and non-tumor tissue samples from 66 ESCC patients and in plasma from ESCC 
A

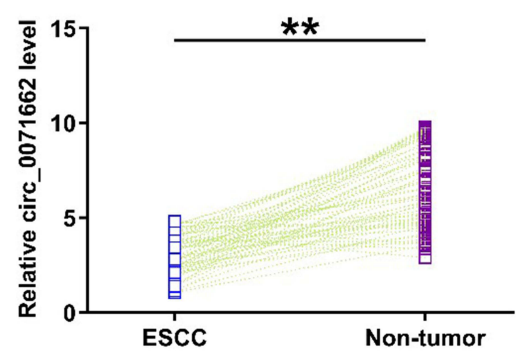

B

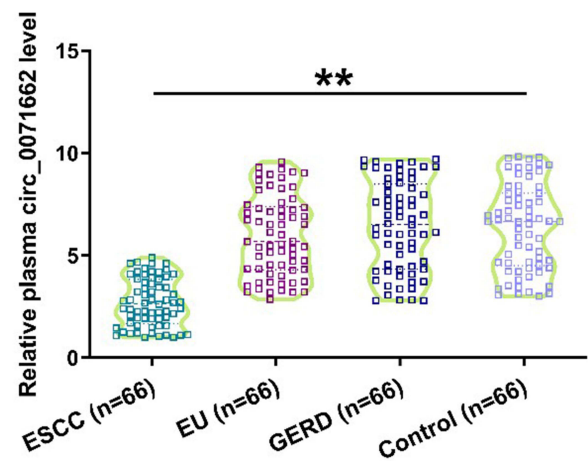

Figure I RT-qPCR analysis of t circ_007/662 levels in tissues and plasma. RNA samples were isolated from paired ESCC and non-tumor tissue samples from 66 ESCC patients $(\mathbf{A})$ and plasma samples from ESCC patients $(n=66)$, EU patients $(n=66)$, GERD patients $(n=66)$, and controls $(n=66)(B)$, and subjected to cDNA synthesis and subsequent qPCR to determine the expression of circ_0071662Circ_0071662 levels. ${ }^{* *} p<0.01$.

patients $(\mathrm{n}=66)$, EU patients $(\mathrm{n}=66)$, GERD patients $(\mathrm{n}=$ 66) and controls $(n=66)$ were determined using RT-qPCR. The results showed circ_0071662 levels were decreased in ESCC tissues than in non-tumor tissues (Figure 1A, p < 0.01), and the plasma circ_0071662 levels also decreased in ESCC patients, but not in GERN and EU patients compared with the healthy controls (Figure 1B, $\mathrm{p}<0.01$ ).

\section{Associations Between Plasma Circ_007I 662 and Patients' Clinical Factors}

The 66 ESCC patients were assigned into high and low plasma circ_0071662 level groups, and chi-squared test was applied to explore the associations between plasma circ_0071662 and patients' clinical factors. Interestingly, plasma circ_0071662 was closely correlated with patients' tumor size but not other clinical features (Table 1). Therefore, it is reasonable to hypothesize that circ_0071662 is mainly involved in the growth of ESCC tumors.

\section{Diagnostic Values of Plasma Circulating Circ_007I662 for ESCC}

With ESCC patients as the true positive cases and other participants (EU, GERD, or controls) as the true negative cases, ROC curve analysis was carried out to analyze the role of plasma circulating circ_0071662 in the diagnosis of

Table I Associations Between Plasma Circ_007I662 and Patients' Clinical Factors

\begin{tabular}{|c|c|c|c|c|c|}
\hline \multicolumn{2}{|l|}{ Clinical Parameters } & \multirow{2}{*}{$\begin{array}{c}\text { Cases } \\
32\end{array}$} & \multirow{2}{*}{$\frac{\text { Low }(n=33)}{15}$} & \multirow{2}{*}{$\frac{\text { High }(\mathrm{n}=\mathbf{3 3})}{17}$} & \multirow{2}{*}{$\frac{\text { P value }}{0.62}$} \\
\hline Age & $<55 y$ & & & & \\
\hline & $>55 y$ & 34 & 18 & 16 & \\
\hline \multirow[t]{2}{*}{ Gender } & Male & 43 & 20 & 23 & 0.44 \\
\hline & Female & 23 & 13 & 10 & \\
\hline \multirow[t]{2}{*}{ Differentiation grade } & Well/Moderate & 48 & 22 & 26 & 0.27 \\
\hline & Poor & 18 & 11 & 7 & \\
\hline \multirow[t]{2}{*}{ Tumor size } & $>5 \mathrm{~cm}$ & 38 & 13 & 25 & 0.62 \\
\hline & $\leq 5 \mathrm{~cm}$ & 28 & 20 & 8 & \\
\hline \multirow[t]{2}{*}{ Primary tumor invasion depth } & $\mathrm{TI} / \mathrm{T} 2$ & 36 & 20 & 16 & 0.003 \\
\hline & $\mathrm{T} 3 / \mathrm{T} 4$ & 30 & 13 & 17 & \\
\hline \multirow[t]{2}{*}{ Lymph node } & Negative & 43 & 20 & 23 & 0.44 \\
\hline & Positive & 23 & 13 & 10 & \\
\hline \multirow[t]{2}{*}{ TNM stage } & $\mathrm{I} / \mathrm{II}$ & 44 & 24 & 20 & 0.30 \\
\hline & III & 22 & 9 & 13 & \\
\hline \multirow[t]{2}{*}{ Smoking } & Yes & 40 & 22 & 18 & 0.31 \\
\hline & No & 26 & 11 & 15 & \\
\hline \multirow[t]{2}{*}{ Drinking } & Yes & 43 & 20 & 23 & 0.44 \\
\hline & No & 23 & 13 & 10 & \\
\hline
\end{tabular}


ESCC. Our data analysis revealed that decreased circ_0071662 plasma levels could be used to effectively separate ESCC patients from GERN patients (Figure 2A), EU patients (Figure 2B) and healthy controls (Figure 2C). Therefore, decreased plasma circ_0071662 may be applied in clinical practice to increase the diagnostic accuracy of ESCC.

\section{Analysis of the Role of Plasma Circulating Circ_007I662 in Predicting the Survival Outcomes of ESCC Patients}

The 66 ESCC patients were grouped into high and low plasma circ_0071662 level groups. The survival curves of both circ_0071662 level groups were plotted based on the 5-year survival data and compared using Log rank test. Low plasma circ_0071662 levels were closely correlated with worse survival outcomes (Figure 3).

\section{Discussion}

The present study mainly analyzed the role of circ_0071662 in ESCC. We showed that circ_0071662 was lowly expressed in ESCC but not in EU and GERD. Moreover, decreased plasma circ_0071662 levels could be used to assist the diagnosis and prognosis of ESCC.
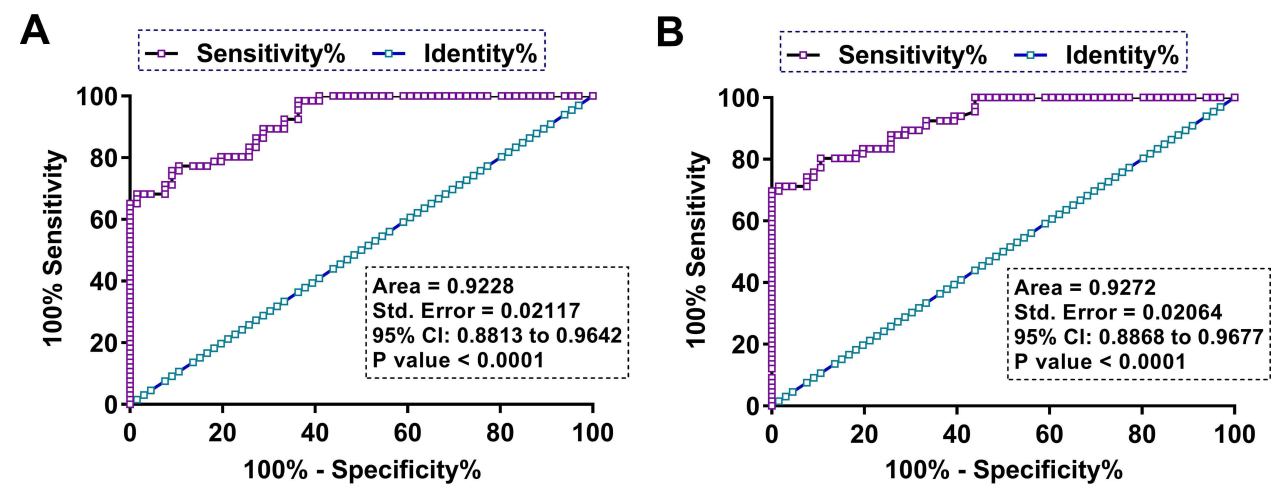

C

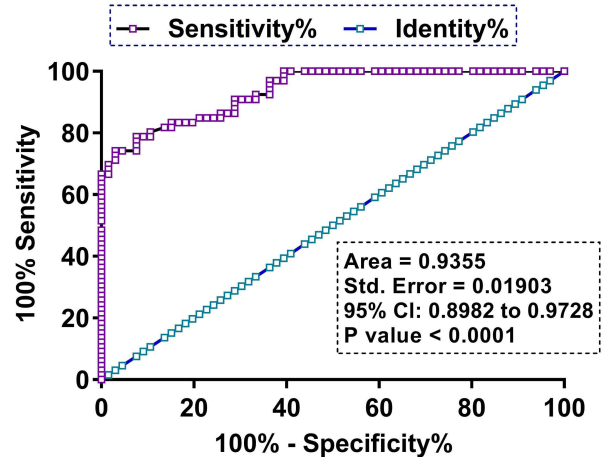

Figure 2 Diagnostic values of plasma circulating circ_007I662 for ESCC. ROC curve analysis was carried out with ESCC patients as the true positive cases and EU (A), GERD (B), or controls (C) as the true negative cases to analyze the role of plasma circulating circ_007I662 in the diagnosis of ESCC.

tumor suppressor in bladder cancer, in which circ_0071662 was found to be lowly expressed and could sponge miR$146 \mathrm{~b}-3 \mathrm{p}$ to suppress its role, thereby decreasing tumor growth and metastasis. ${ }^{15}$ To our best knowledge, the involvement of circ_0071662 in other cancers is unclear. The present study showed that circ_0071662 is specifically downregulated in ESCC but not in EC and GERD, compared to the healthy controls. Therefore, circ_0071662 may be specifically involved in ESCC. Although we did not analyze the role of circ_0071662 in the development and progression of ESCC, we showed that plasma circ_0071662 level is only closely correlated with ESCC tumor size, but not ESCC tumor differentiation and invasion. Therefore, circ_0071662 is likely only involved in the growth, but not metastasis, of ESCC tumors. However, our speculation is purely based on statistical analysis. Future studies with in vitro cell and/or in vivo animal studies are needed to further confirm our conclusions.

In clinical practices, ESCC is frequently misdiagnosed as EU and GERD, which can mimic the symptoms of ESCC, leading to delayed diagnosis and the development of tumor metastasis. The present study showed that decreased plasma circ_0071662 levels 


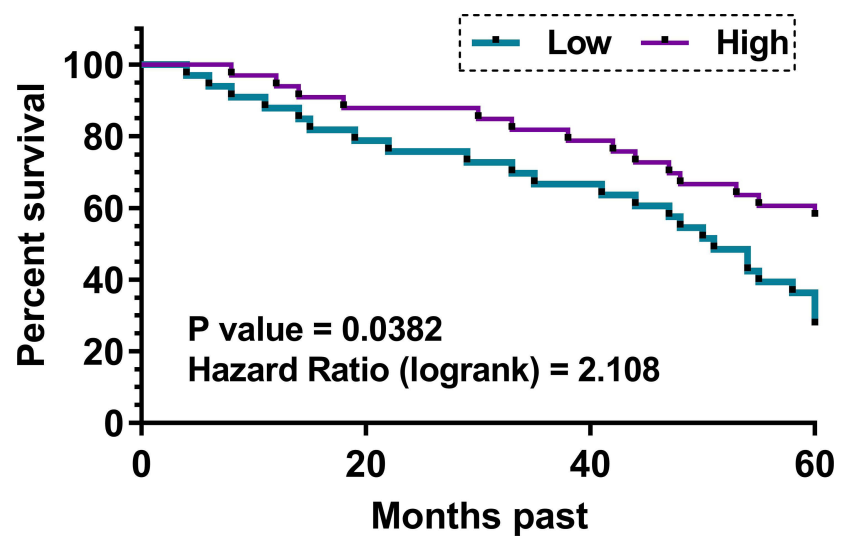

Figure 3 Analysis of the role of plasma circulating circ_0071662 in predicting the survival outcomes of ESCC patients. The 66 ESCC patients were grouped into high and low plasma circ_0071662 level groups. The survival curves of both circ_0071662 level groups were plotted using the 5-year survival data and compared using the Log rank test.

could effectively separate ESCC patients from EU and GERD patients. Therefore, measuring plasma circ_0071662 levels prior to treatment may increase the diagnostic accuracy of ESCC. Moreover, plasma circ_0071662 was found to be closely correlated with the worse survival outcomes of ESCC patients. Our data suggested that plasma circ_0071662 could be used in clinical practices to identify patients with a high risk of death, thereby designing personalized treatment approaches to prolong survival. Although previous studies have reported the role of circRNAs as biomarkers for ESCC, these studies failed to include other diseases that can mimic the symptoms of ESCC. ${ }^{16,17}$ Our study is the first to report the application of a circRNA to distinguish ESCC from EU and GERD.

This study is limited by the small sample size. Our conclusions should be verified by future studies. In addition, functional assays are needed to explore the function of circ_0071662 in ESCC.

\section{Conclusion}

Circ_0071662 is lowly expressed in ESCC and decreased plasma circ_0071662 may serve as a potential diagnostic biomarker to separate ESCC patients from EU and GERD patients. In addition, patients with low circ_0071662 levels may have poor prognoses.

\section{Data Sharing Statement}

The analyzed data sets generated during the study are available from the corresponding author on reasonable request.

\section{Ethical Approval and Consent to Participate}

All patients signed the written informed consent. All procedures were approved by the Ethics Committee of the Third Affiliated Hospital of Chongqing Medical University and completed in keeping with the standards set out in the Announcement of Helsinki and the Laboratory Guidelines of Research in China.

\section{Acknowledgments}

We thank the financial support from the Chongqing Science and Health Combined Medical Research Project (No. 2019MSXM024).

\section{Funding}

The study was supported by the Chongqing Science and Health Combined Medical Research Project (No. 2019MSXM024).

\section{Disclosure}

The authors declare that they have no conflicts of interest.

\section{References}

1. Abnet CC, Arnold M, Wei WQ. Epidemiology of esophageal squamous cell carcinoma. Gastroenterology. 2018;154(2):360-373. doi:10.1053/j.gastro.2017.08.023

2. Codipilly DC, Qin Y, Dawsey SM, et al. Screening for esophageal squamous cell carcinoma: recent advances. Gastrointest Endosc. 2018;88(3):413-426. doi:10.1016/j.gie.2018.04.2352

3. Murphy G, McCormack V, Abedi-Ardekani B, et al. International cancer seminars: a focus on esophageal squamous cell carcinoma. Ann Oncol. 2017;28(9):2086-2093. doi:10.1093/annonc/mdx279

4. Wang Q, Wang F, Lv J, et al. Interactive online consensus survival tool for esophageal squamous cell carcinoma prognosis analysis. Oncol Lett. 2019;18(2):1199-1206. doi:10.3892/ol.2019.10440

5. Then EO, Lopez M, Saleem S, et al. Esophageal cancer: an updated surveillance epidemiology and end results database analysis. World J Oncol. 2020;11(2):55-64. doi:10.14740/wjon1254

6. Shaheen O, Ghibour A, Alsaid B. Esophageal cancer metastases to unexpected sites: a systematic review. Gastroenterol Res Pract. 2017;2017:1657310. doi:10.1155/2017/1657310

7. Visaggi P, Barberio B, Ghisa M, et al. Modern diagnosis of early esophageal cancer: from blood biomarkers to advanced endoscopy and artificial intelligence. Cancers. 2021;13(13):3162. doi:10.3390/ cancers 13133162

8. Chadwick G, Groene O, Hoare J, et al. A population-based, retrospective, cohort study of esophageal cancer missed at endoscopy. Endoscopy. 2014;46(7):553-560. doi:10.1055/s-0034-1365646

9. Horie Y, Yoshio T, Aoyama K, et al. Diagnostic outcomes of esophageal cancer by artificial intelligence using convolutional neural networks. Gastrointest Endosc. 2019;89(1):25-32. doi:10.1016/j. gie.2018.07.037

10. Sasaki Y, Suzuki T, Zai H, Urita Y. Esophageal ulcer associated with inappropriately taken doxycycline: a benign mimicker of esophageal cancer. J Gen Fam Med. 2017;18(4):171-172. doi:10.1002/jgf2.55

11. Hao J, Liu B, Yang CS, Chen X. Gastroesophageal reflux leads to esophageal cancer in a surgical model with mice. $B M C$ Gastroenterol. 2009;9:59. doi:10.1186/1471-230X-9-59 
12. Wu SG, Zhang WW, He ZY, Sun JY, Chen YX, Guo L. Sites of metastasis and overall survival in esophageal cancer: a population-based study. Cancer Manag Res. 2017;9:781-788. doi:10.2147/CMAR.S150350

13. Huang X, Zhou X, Hu Q, et al. Advances in esophageal cancer: a new perspective on pathogenesis associated with long non-coding RNAs. Cancer Lett. 2018;413:94-101. doi:10.1016/j.canlet.2017. 10.046

14. Niu C, Zhao L, Guo X, Shen Y, Shao Y, Liu F. Diagnostic accuracy of circRNAs in esophageal cancer: a meta-analysis. Dis Markers. 2019;2019:9673129. doi:10.1155/2019/9673129
15. Abulizi R, Li B, Zhang CG. Circ 0071662, a novel tumor biomarker, suppresses bladder cancer cell proliferation and invasion by sponging miR-146b-3p. Oncol Res. 2019. doi:10.3727/096504019X1 5740729375088

16. Guo C, Mi J, Li H, et al. Dysregulated circular RNAs as novel biomarkers in esophageal squamous cell carcinoma: a meta-analysis. Cancer Med. 2021. doi:10.1002/cam4.3703

17. Zhang X, Lu N, Wang L, et al. Circular RNAs and esophageal cancer. Cancer Cell Int. 2020;20(1):362. doi:10.1186/s12935-020-01451-0

\section{Publish your work in this journal}

The International Journal of General Medicine is an international, peer-reviewed open-access journal that focuses on general and internal medicine, pathogenesis, epidemiology, diagnosis, monitoring and treatment protocols. The journal is characterized by the rapid reporting of reviews, original research and clinical studies across all disease areas. The manuscript management system is completely online and includes a very quick and fair peer-review system, which is all easy to use. Visit http://www.dovepress.com/ testimonials.php to read real quotes from published authors. 